\title{
Unveiling the dual role of the cholinium hexanoate ionic liquid as solvent and catalyst in suberin depolymerisation $\dagger$
}

\author{
Rui Ferreira, $\ddagger^{\mathrm{a}}$ Helga Garcia, $\dot{\dagger}^{\mathrm{a}}$ Andreia F. Sousa, ${ }^{\mathrm{bc}}$ Marina Guerreiro, ${ }^{\mathrm{a}}$ \\ Filipe J. S. Duarte, ${ }^{\mathrm{d}}$ Carmen S. R. Freire, ${ }^{\mathrm{b}}$ Maria José Calhorda,

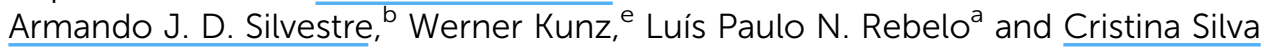 \\ Pereira*a
}

\begin{abstract}
Disruption of the three-dimensional network of suberin in cork by cholinium hexanoate leads to its efficient and selective isolation. The reaction mechanism, which likely involves selective cleavage of some intermonomeric bonds in suberin, was still unanswered. To address this question, the role of the ionic liquid during suberin depolymerisation and during cleavage of standard compounds carrying key chemical functionalities was herein investigated. A clear demonstration that the ionic liquid catalyses the hydrolysis of acylglycerol ester bonds was attained herein, both experimentally and computationally (DFT calculations). This behaviour is related to cholinium hexanoate capacity to activate the nucleophilic attack of water. The data showed also that the most favourable reaction is the hydrolysis of acylglycerol ester bonds, with the C2 position reporting the faster kinetics, whilst most of the linear aliphatic esters remained intact. The study emphasises that the ionic liquid plays the dual role of solvent and catalyst and leads to suberin efficient extraction through a mild depolymerisation. It is also one of the few reports of ionic liquids as efficient catalysts in the hydrolysis of esters.
\end{abstract}

Received 17th October 2013

Accepted 18th November 2013

DOI: 10.1039/c3ra45910a

www.rsc.org/advances

\section{Introduction}

Renewable resources are increasingly regarded as substituents for highly demanded petroleum-based chemicals. ${ }^{1}$ In this context, the extraction, application and biosynthesis of plant biopolyesters, namely cutin and suberin, have been extensively studied. ${ }^{2}$ Suberin is ubiquitous in higher plants, and particularly abundant in the outer barks of Quercus suber and Betula pendula and in the peel of Solanum tuberosum. This hydrophobic polymeric material is deposited in the secondary cell wall in the internal and the peripheral dermal tissues during cell wall differentiation or as a response to stress and wounding.

\footnotetext{
anstituto de Tecnologia Quimica e Biológica, Universidade Nova de Lisboa, Apartado 127, 2781 901, Oeiras, Portugal. E mail: spereira@itqb.unl.pt

${ }^{b}$ CICECO and Department of Chemistry, University of Aveiro, Campus de Santiago, 3810193 Aveiro, Portugal

'Department of Chemical Engineering, University of Coimbra, Pólo II, Pinhal de Marrocos, 3030290 Coimbra, Portugal

${ }^{d}$ Departamento de Quimica e Bioquimica, CQB, Faculdade de Ciências, Universidade de Lisboa, 1749016 Lisboa, Portugal

'Institute of Physical and Theoretical Chemistry, University of Regensburg, D 93040 Regensburg, Germany

$\dagger$ Electronic supplementary information (ESI) available: Fig. S1 (thermal microscopy analysis); Table S1 (GC MS data) and S3 (cartesian coordinates and computed total energies). See DOI: 10.1039/c3ra45910a

\$ Equally contributing authors.
}

Suberin builds an apoplastic barrier that controls the flow of water, gases and ions and protects against biological pathogens and physical aggressions. ${ }^{911}$

Since the earliest descriptions, suberin is known to be composed of aromatic and aliphatic monomeric units. ${ }^{12}{ }^{14}$ Currently, the prevailing idea is that suberin comprises two covalently linked domains, the major being the polyaliphatic domain and the minor the polyphenolic one. ${ }^{10,15}{ }^{18}$ Suberin is generally accepted to be organised in situ as a lamellar type structure, ${ }^{16}{ }^{19}$ notwithstanding that some structural aspects are still unanswered. The polyphenolic domain is ingrained on the inner face of the primary cell wall. ${ }^{10,16}{ }^{18,20,21}$ Especially due to its high recalcitrancy, the compositional structure of this ligninlike domain is not yet fully understood. ${ }^{22,23}$ Even so, it is known to be composed mainly by hydroxycinnamic acids and their derivatives (predominantly ferulic acid) and some vestigial amounts of monolignols (viz. $p$-coumaryl, coniferyl and sinapyl alcohols). Extensive cross-linking between these aromatic monomers in the lignin-like structure, as well as to other cell wall constituents, is done via stable carbon-carbon, amide and ether bonds. ${ }^{9,23} 25$

The polyaliphatic domain is composed mostly of long chains $\left(\mathrm{C}_{16}-\mathrm{C}_{26}\right)$ of alkanols, alkanoic acids, $\omega$-hydroxyalkanoic acids and $\alpha, \omega$-alkanedioic acids (and the corresponding mid-chain unsaturated, epoxy or vic-diol derivatives) and glycerol. In addition, the deposition of hydroxycinnamates leads to the 
typical lamellar organisation of suberin of alternate aliphatic and phenolic components. ${ }^{16}$ These monomers are in a parallel alignment and linked via linear aliphatic ester or acylglycerol ester bonds. Glycerol is a key cross-linker in the formation of a three-dimensional network, connecting hydrophilic moieties and both suberin domains. ${ }^{\mathbf{1 6 , 2 5}}$

Knowledge on suberin is spread in diverse scientific disciplines, from biology, e.g. the understanding of the polymer biosynthesis, ${ }^{\mathbf{4}, 7}$ to chemistry, e.g. the depolymerisation and characterisation of suberin. ${ }^{17}$ Although only modestly exploited so far, suberin has been regarded as a source of monomers and oligomers for the synthesis of novel macromolecular materials.9,26 29 Of particular relevance is the abundance of mid-chain hydroxy and epoxy fatty acids in suberin. These are rare in other renewable resources and difficult to synthesise chemically.

Suberin extraction from renewable resources is conventionally attained using alkaline hydrolysis ${ }^{30}$ or alkaline methanolysis. $^{17,31}$ These methods result in extensive ester bond cleavage, i.e. depolymerisation. Although with very low extraction efficiency, comparable methods can be used to promote a partial depolymerisation. ${ }^{17}$ An alternative extraction method, using cholinium hexanoate, promotes efficient extraction of a still partially cross-linked and highly polymerised suberin, regardless of the renewable resource used, viz. cork, ${ }^{32}{ }^{34}$ birch outer bark ${ }^{34}$ and potato peel (unpublished work). The uniqueness of such method is highlighted by the compositional structure of suberin, hypothesised to be intimately related to that of the in situ suberin. ${ }^{32}$ Emphasis should be also given to the high biocompatibility and biodegradability of the ionic liquid used in the extraction, ${ }^{35}$ which can be recycled and reused throughout the process. ${ }^{32}$

Suberin depolymerisation induced by cholinium hexanoate is likely to involve the selective cleavage of some bonds linking suberin composing monomers. This is the underlying question of the present study: to solve the chemical reaction mechanism behind suberin depolymerisation in cholinium hexanoate media. To address this question, we further investigated the lability of key chemical functionalities of suberin and of standard compounds in the ionic liquid media. The chemical mechanism proposed herein was also supported by DFT calculations.

\section{Experimental}

Cork

Granulated cork was obtained from the cork producers Amorim \& Irmãos SA (Santa Maria de Lamas, Portugal). The samples were ground to a fine powder (60 mesh) using a centrifuge mill (Retsch) and the cork extractives removed by sequential Soxhlet extraction with solvents of increasing polarity (dichloromethane, ethanol and water) as previously described by Gil et $a l^{36}$ The extractive-free cork powder, hereinafter defined solely as cork, was further washed with an excess of deionised water for complete removal of low molecular weight compounds, and dried prior to use.

\section{Chemicals}

Cholinium hexanoate was synthesised by dropwise addition of hexanoic acid to aqueous cholinium hydrogen carbonate (Sigma $\sim 80 \%$ in water) in equimolar quantities, as previously described. ${ }^{35}$ The ionic liquid purity was verified by ${ }^{1} \mathrm{H}$ - and ${ }^{13} \mathrm{C}$ NMR, CHNS elemental analysis and electrospray ionisation mass spectrometry (ESI-MS). The ionic liquid was dried prior to use by stir-heating in vacuum $\left(40-50{ }^{\circ} \mathrm{C}, c a .0 .01 \mathrm{mbar}\right)$. The water content, determined by Karl-Fischer titration, was ca. $0.2 \mathrm{wt} \%$.

Poly(12-hydroxydodecanedioic acid) was prepared as described before ${ }^{29}$ and 9-10-epoxy-18-hydroxyoctadecanoic acid was extracted from Betula pendula. ${ }^{37}$

Octyl octanoate $(\geq 98 \%)$, glyceryl trioctanoate $(\geq 99 \%)$, dimethyl sulfoxide (DMSO, 99.5\%), sodium hydroxide (>97\%) and dichloromethane $(\geq 99.5 \%)$ were purchased from Sigma; glycerol ( $\geq 99.5 \%)$ from VWR, L-lactic acid (99.5\%) from Fluka and poly(lactic acid) with an L : D ratio of $96: 4$ and an $M_{\mathrm{w}}$ of $110000 \mathrm{~g} \mathrm{~mol}^{-1}$ from Cargill-Dow Polymers.

\section{Suberin extraction}

The suberin extraction process followed a methodology which has been previously described. ${ }^{32,34}$ Briefly, the cholinium hexanoate (melting temperature, $60.57{ }^{\circ} \mathrm{C}$ ) was mixed with cork (ionic liquid : cork $\approx 9: 1 \mathrm{wt} / \mathrm{wt}$ ) and kept at $100{ }^{\circ} \mathrm{C}$ during 1,2 , 4 or 8 hours, with stirring (each in triplicate). At the end of the extraction process, DMSO was added to reduce the viscosity of the mixture, ${ }^{32}$ facilitating its filtration through a nylon membrane with an exclusion pore size of $1.0 \mu \mathrm{m}$ (Millipore, MA, USA). The insoluble residue was then washed thoroughly with an excess of water at $80{ }^{\circ} \mathrm{C}$. Precipitation of the extracted suberin was obtained by keeping the ensuing filtrate (i.e. ionic liquid, suberin, DMSO and the water added to wash the insoluble residue) at $4{ }^{\circ} \mathrm{C}$ for 1 hour. Suberin was then recovered by centrifugation ( $30 \mathrm{~min}$ at $4{ }^{\circ} \mathrm{C}$ and $2450 \times \mathrm{g}$ ), washed twice with an excess of water and dried at $50{ }^{\circ} \mathrm{C}$, until constant weight was attained. The aqueous phase (supernatant) was also concentrated in a rotary evaporator and its content in glycerol analysed by high performance liquid chromatography (HPLC).

\section{Attenuated total reflectance-Fourier transform infrared spectroscopy (ATR-FTIR)}

ATR-FTIR spectra were collected on a Bruker IFS66/S FTIR spectrometer (Bruker Daltonics, MA, USA) using a single reflection ATR cell (DuraDisk, equipped with a diamond crystal). Data were recorded at room temperature, in the range of $4000-600 \mathrm{~cm}^{-1}$, by accumulating 128 scans with a resolution of $4 \mathrm{~cm}^{-1}$. Five replica spectra were collected for each sample in order to evaluate reproducibility (OPUS v6.0).

\section{Nuclear magnetic resonance spectroscopy (NMR)}

${ }^{1} \mathrm{H}$ - and ${ }^{13} \mathrm{C}-\mathrm{NMR}$ analyses were recorded with a Bruker Avance 400 Ultrashield Plus spectrometer. Spectra were run at $25{ }^{\circ} \mathrm{C}$ using standard Bruker pulse programs. ${ }^{13} \mathrm{C}$ CP/MAS NMR spectra were recorded at $9.4 \mathrm{~T}$ on a Bruker 400 spectrometer 
using $9 \mathrm{kHz}$ spinning rate and MAS with proton $90^{\circ}$ pulses of $4 \mu \mathrm{s}$. Chemical shifts are given in ppm from glycine. The NMR spectra were processed and analysed with MestreNova v6.0 (MestreLab Research S.L.).

\section{Gas chromatography-mass spectrometry (GC-MS)}

A Trace GC 2000 Series gas chromatograph equipped with a Thermo Scientific DSQ II mass spectrometer was used. The GCMS was first calibrated with pure reference compounds (representative of the major classes of compounds present in suberin) relative to $n$-hexadecane (internal standard). Compounds identification was based on the equipment spectral library (Wiley-Nist) and on previously published data, focussing their EI-MS fragmentation patterns and/or retention times. ${ }^{30,31,38,39}$ Replicates were done to guarantee low variability and each analysis repeated twice. Each suberin sample was analysed by two complementary methods:

- Method 1, samples were converted to the corresponding trimethylsilyl (TMS) derivatives and analysed by GC-MS. In brief, suberin samples ( $c a .15 \mathrm{mg}$ ) were reacted with $250 \mu \mathrm{L}$ of $N, O$-bis(trimethylsilyl)trifluoroacetamide and $50 \mu \mathrm{L}$ of trimethylchlorosilane in $250 \mu \mathrm{L}$ of pyridine, during $30 \mathrm{~min}$ at $70^{\circ} \mathrm{C}^{40}$

- Method 2, samples were submitted to alkaline hydrolysis prior to the silylation, to release hydrolysable monomeric constituents. Briefly, suberin samples were treated with a solution of $0.5 \mathrm{M} \mathrm{NaOH}$ in methanol/water $(1: 1, \mathrm{v} / \mathrm{v})$ at $95{ }^{\circ} \mathrm{C}$, during 4 hours..$^{41}$ The mixture was cooled to room temperature, acidified to $\mathrm{pH} 3-3.5$ with $1 \mathrm{M} \mathrm{HCl}$, and extracted three times by dichloromethane/water partition. The combined organic extracts were dried in a rotary evaporator, then trimethylsilylated as mentioned above, and analysed by GC-MS. The aqueous phases were also concentrated in a rotary evaporator and their content in glycerol analysed by HPLC.

\section{High performance liquid chromatography}

Samples were analysed by HPLC, using a Waters chromatographer, consisting of a 510 Pump, a 715 Autosampler and a Temperature Control Module (Waters Chromatography, Milford, MA, USA), connected to a LKB 2142 Differential Refractometer detector (Bromma, Sweden). Data acquisition was accomplished with the Millenium32 v3.05.01, 1998 system (Waters). Chromatographic separation was undertaken at $60^{\circ} \mathrm{C}$ using an Aminex HPX-87H column $(300 \times 7.8 \mathrm{~mm})$ with $9 \mu \mathrm{m}$ particle size (Bio-Rad, Hercules, California). Elution was carried out isocratically, at a flow rate of $0.5 \mathrm{~mL} \mathrm{~min}{ }^{-1}$, with $0.005 \mathrm{~N}$ of $\mathrm{H}_{2} \mathrm{SO}_{4}$ and the sample volume injected was $20 \mu \mathrm{L}$. Lactic acid (retention time $=15.98 \mathrm{~min}$ ) and glycerol (retention time $=15.20 \mathrm{~min}$ ) quantifications were done using an external calibration curve with high purity standards and within the quantification limits of $0.25-20.41 \mathrm{mg} \mathrm{mL}^{-1}$ and $0.25-9.99 \mathrm{mg} \mathrm{mL}^{-1}$, respectively.

\section{Reaction of standard compounds in cholinium hexanoate media}

Tests of cholinium hexanoate ability to cleave standard compounds were done in the same conditions used for cork, i.e.
$100{ }^{\circ} \mathrm{C}$, with stirring (experimental triplicates and technical duplicates).

Glyceryl trioctanoate and octyl octanoate were mixed with cholinium hexanoate at a 1.5 ratio of ionic liquid moles per mole of ester bonds, during 1, 2, 4 or 8 hours. At the end of the test, after partition of the ensuing mixture in dichloromethane/ water, the organic layer was recovered, concentrated in a rotary evaporator, dried at $50{ }^{\circ} \mathrm{C}$, and then analysed by GC-MS as described in GC-MS - Method 1. The aqueous phases were also concentrated in a rotary evaporator and their content in glycerol analysed by HPLC.

The remaining standard compounds, namely 9,10-epoxy-18hydroxyoctadecanoic acid, poly(12-hydroxydodecanoic acid) and poly(lactic acid) were mixed with cholinium hexanoate for 4 hours, using a mass ratio (standard: cholinium hexanoate) of $1: 4,1: 3,1: 10,1: 10$ and $1: 10$, respectively. For the first two compounds, at the end of the test, an excess of water was added, the precipitate recovered by centrifugation, dried and analysed by ATR-FTIR and/or NMR. In the test with poly(lactic acid), after the reaction, an excess of water was added, the insoluble residue recovered by filtration, dried and then analysed by ATR-FTIR. The content of lactic acid in the aqueous phase was determined by HPLC.

\section{Thermal microscopy}

Thermal microscopy analyses were done using a Leitz Orthoplan polarizing microscope (Wetzlar, Germany) equipped with a JVC digital camera (TK-C130), a Linkham hot stage with a TMS90 temperature controller $\left( \pm 0.5{ }^{\circ} \mathrm{C}\right)$ and a CS196 cooling system.

\section{Computational methods}

Density functional theory (DFT ${ }^{42,43}$ calculations were performed with the Gaussian 09 software package ${ }^{44}$ with the hybrid functional PBE1PBE ${ }^{45}{ }^{48}$ and the $6-31+\mathrm{G}^{* *}$ basis set. Full geometry optimisations including solvent effects (DMSO) were carried out, using the polarisable continuum model (PCM) ${ }^{49}$ The starting structures are based in the most stable approach geometry of the ionic liquid and reactants. Harmonic vibrational frequencies were calculated for all located stationary structures to verify whether they were minima or transition states. Zero-point energies and thermal corrections were taken from unscaled vibrational frequencies. Free energies of activation are given at $25{ }^{\circ} \mathrm{C}$. All energies were calculated relative to the reagents. All bond lengths are in angstrom $(\AA)$ and energies in $\mathrm{kJ} \mathrm{mol}^{-1}$.

\section{Results and discussion}

The cholinium-hexanoate-based method for suberin extraction combines partial depolymerisation of suberin with high selectivity and extraction efficiency. ${ }^{32}{ }^{34}$ To identify the chemical bonds prone to be cleaved in the cholinium hexanoate media, we carried out a comprehensive study of the lability of suberin and of standard compounds carrying the key structural bonds 
in suberin, namely linear aliphatic esters and acylglycerol esters, as depicted schematically in Fig. 1.

\section{Suberin depolymerisation occurs through cleavage of ester bonds: time-course analysis}

The natural abundance of suberin in cork is typically ca. 50-60 wt $\%{ }^{10}$ The extraction of suberin from cork with cholinium hexanoate was extremely fast, given that after one hour the recovery yield was $46.8 \mathrm{wt} \%$. However, after this phase the suberin recovery yield showed a minor increase over time, reaching $54.7 \mathrm{wt} \%$ at the eighth hour of extraction (Table 1).

Thermal microscopy analysis showed a rapid disruption of cork during the first hour of the extraction. No significant differences were observed for longer periods. Suberin extraction was probably facilitated by this disruption, which exposes the cell wall components to the ionic liquid phase (Fig. S1†).

Aiming to identify which chemical bonds in suberin were cleaved in the cholinium hexanoate media, structural composition analyses of the samples recovered along the extraction time were undertaken. After eight hours of extraction, the ester features, namely the resonance at $\delta 173 \mathrm{ppm}\left({ }^{13} \mathrm{C} \mathrm{CP} / \mathrm{MAS} N M R\right.$, Fig. 2A) and the carbonyl stretching at $1731 \mathrm{~cm}^{-1}$ (ATR-FTIR, Fig. 2B), were still detected in the corresponding spectra. The intensity of the ester resonance at $\delta 173 \mathrm{ppm}$ decreased along the extraction time, suggesting ester bond cleavage (ATR-FTIR, Fig. 2B). In addition, the peak at $1715 \mathrm{~cm}^{-1}$, which was barely distinguishable in the first hours of the extraction, for longer periods became a defined peak. This peak is associated with hydrogen bonded carbonyl groups in either esters or acids (see magnification in Fig. 2B), corroborating the idea that ester bond cleavage occurred. It becomes apparent that the ionic liquid led to mild depolymerisation of suberin through continuous ester bond cleavage.

Other important observations in the ${ }^{13} \mathrm{C}$ CP/MAS NMR spectra of the recovered suberin samples are the two major resonances at $\delta 33$ and $30 \mathrm{ppm}$ which are associated with aliphatic methylenes (Fig. 2A). The ratio 33/30 ppm increased over the extraction time. The carbons at $\delta 33 \mathrm{ppm}$ have been suggested to report slightly lower mobility in comparison to those at $\delta 30 \mathrm{ppm} .^{31}$ This has been related to the presence of methylene groups near oxygen vicinities (suggestive of linkage to lignin and polysaccharides), ${ }^{31}$ or to the presence of crystalline/recalcitrant domains. ${ }^{50,51}$ Other resonances typical of suberin can be noticed in all spectra, namely signals at $\delta 50-90 \mathrm{ppm}, \delta 130$ and $148 \mathrm{ppm}$, assigned to carbons linked to oxygen, vinylic and quaternary carbons, respectively. One cannot disregard the hypothesis that cholinium hexanoate contamination contributed to the resonance at $\delta 54 \mathrm{ppm}$ (more intense in the suberin samples extracted for one or two hours, see * in Fig. 2A).

The ATR-FTIR spectra of the suberin samples show two major peaks at 2918, $2851 \mathrm{~cm}^{-1}$ and a band between 3679 and $3034 \mathrm{~cm}^{-1}$ (Fig. 2B). These are respectively associated to the aliphatic chains and to the hydroxyl groups in carboxylic acids and/or alcohols. The hydroxyl band increased along the extraction time. This can be related to the release of hydroxyl moieties, which reinforce the idea that suberin depolymerisation was continuous along the course of the extraction.

More information on the monomeric composition of suberin along the extraction time can be retrieved using GC-MS analysis. The underlying concept here is that suberin extracted with cholinium hexanoate is still cross-linked and/or oligomeric. Consequently, the high molecular weight of these structures hampers their detection by GC-MS (see GC-MS - Method 1, Experimental section). In agreement, very low identification yields were attained ( $c a .2-5 \mathrm{wt} \%$ ), notwithstanding that the main typical suberin monomers could be detected (data not shown). Monoacylglycerol dimers were only detected in the suberin samples recovered after the first two hours of the extraction, reinforcing the idea that, until the end of the extraction time, oligomers released to the ionic liquid media were further depolymerised.

Aiming to overcome the low GC-MS identification yields, any hydrolysable chemical bonds in the ionic liquid extracted suberin were cleaved by conventional alkaline hydrolysis before the analysis (see GC-MS - Method 2, Experimental section). Consequently, the identification yields of these samples were significantly higher, $c a$. $50 \mathrm{wt} \%$ (Table $\mathrm{S} 1 \dagger$ ). In general, the

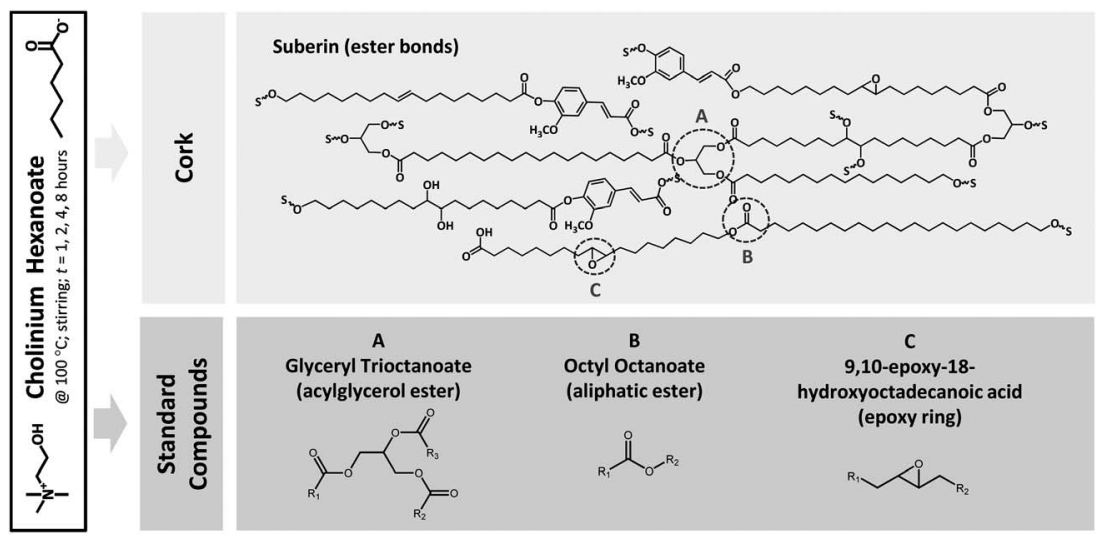

Fig. 1 Schematic view of the experiments undertaken to determine the susceptibility of ester bonds and epoxy rings to undergo cleavage in the cholinium hexanoate media. Cholinium hexanoate tests with cork (top) and with standard compounds (bottom). The standard compounds reproduce main chemical functionalities present in suberin, namely acylglycerol ester bonds (A), aliphatic ester bonds (B) and epoxy rings (C). 
Table 1 Analysis of suberin extracted from cork by cholinium hexanoate. Values depicted correspond to suberin recovery yield $\left[100\left(m_{\text {recovered suberin }} / m_{\text {cork }}\right)\right]$, solubility in dichloromethane $\left[100\left(m_{\text {soluble suberin }} / m_{\text {suberin }}\right)\right]$ and alkaline hydrolysis recalcitrancy $\left[100 \quad\left(100\left(m_{\text {hydrolysable }}\right.\right.\right.$ suberin $\left.\left./ m_{\text {suberin }}\right)\right]$ and to the quantification of the glycerol released during suberin depolymerisation, namely in the cholinium hexanoate media

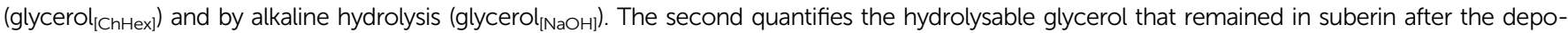
lymerisation of suberin in the ionic liquid media. Values in brackets stand for standard deviation between replicates

\begin{tabular}{|c|c|c|c|c|c|}
\hline Time/h & & 1 & 2 & 4 & 8 \\
\hline Recovery yield/wt\% & & $46.8(4.5)$ & $47.3(0.9)$ & $51.0(5.8)$ & $54.7(14.8)$ \\
\hline Solubility in $\mathrm{CH}_{2} \mathrm{Cl}_{2} / \mathrm{wt} \%$ & & $46.2(0.3)$ & $48.0(1.1)$ & $55.7(2.9)$ & $65.2(1.7)$ \\
\hline Alkaline hydrolysis recalcitrancy/wt\% & & $34.4(3.9)$ & $37.7(12.4)$ & $49.0(7.8)$ & $46.3(0.1)$ \\
\hline \multicolumn{6}{|l|}{ Glycerol $\mathrm{mg}_{\text {glycerol }} / \mathrm{g}_{\text {suberin }}$} \\
\hline \multirow[t]{3}{*}{ Glycerol $_{[\mathrm{ChHex}]}{ }^{a}$} & HPLC & $17.4(3.0)$ & $18.6(3.1)$ & $51.1(4.1)$ & $63.0(21.2)$ \\
\hline & GC MS & $4.3(0.4)$ & $5.5(2.3)$ & $2.8(1.0)$ & $2.7(2.5)$ \\
\hline & Total & 21.7 & 24.1 & 53.9 & 65.7 \\
\hline \multirow{3}{*}{ Glycerol $_{[\mathrm{NaOH}]}^{b}$} & HPLC & $34.9(4.5)$ & $32.2(3.2)$ & $7.6(1.9)$ & $0.0(0.0)$ \\
\hline & GC MS & $0.8(0.3)$ & $0.7(0.2)$ & $0.4(0.3)$ & $0.3(0.1)$ \\
\hline & Total & 35.7 & 32.9 & 8.0 & 0.3 \\
\hline Total glycerol & & 57.4 & 57.0 & 61.9 & 66.0 \\
\hline
\end{tabular}

${ }^{a}$ Glycerol $_{[\mathrm{ChHex}]}$, glycerol released during suberin depolymerisation in the cholinium hexanoate media, calculated as the total of the glycerol detected by HPLC (i.e. solubilised in water during the filtration and the precipitation steps) and by GC MS (i.e. present in the sample). ${ }^{b}$ Glycerol $_{[\mathrm{NaOH}]}$, glycerol released during alkaline hydrolysis of suberin samples, calculated as the total of the glycerol detected by HPLC (i.e. solubilised in the aqueous phase during dichloromethane/water partition after the alkaline hydrolysis) and by GC MS (in the sample after alkaline hydrolysis).
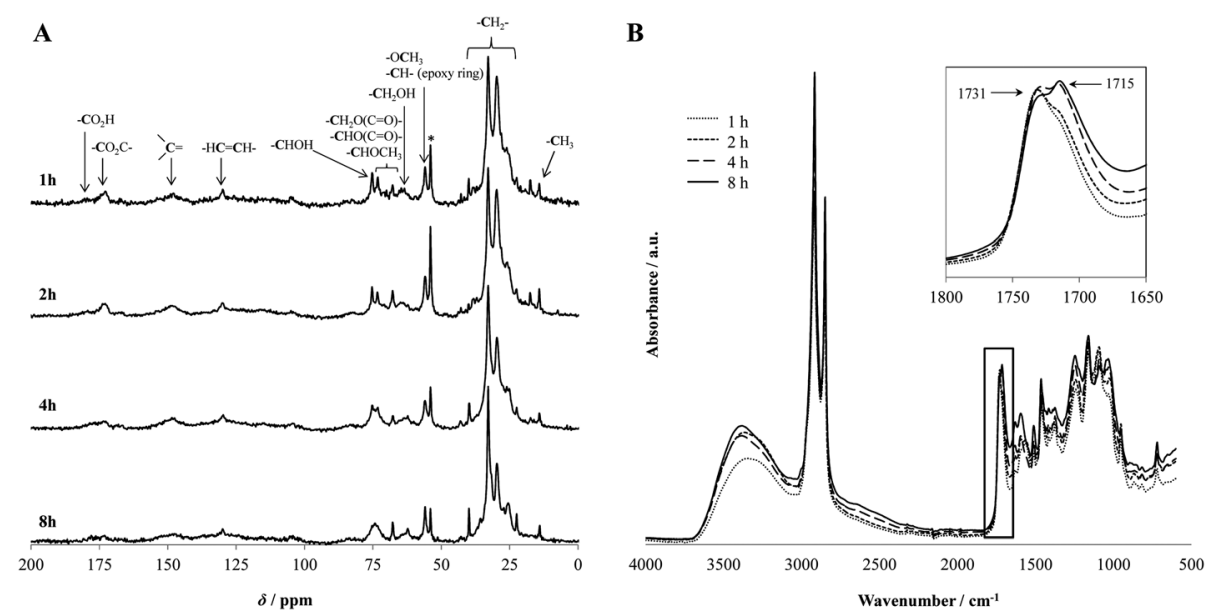

Fig. 2 13C CP/MAS NMR spectra (A) and ATR-FTIR spectra (B) of suberin extracted with cholinium hexanoate after 1, 2, 4 or 8 hours.

amounts of the monomers herein identified were coherent with those typically reported for suberin extracted with conventional methods. ${ }^{17,30,31}$ Major compounds identified were $\omega$-hydroxyalkanoic acids, followed by $\alpha, \omega$-alkanedioic acids and phenolics, and minor ones were alkanoic acids and alkan-1-ols (Fig. 3, Table $\mathrm{S} 1 \dagger)$. Similar to previous reports, ${ }^{17}$ the most abundant monomers herein detected were 22-hydroxydocosanoic acid, followed by ferulic acid, 9,10,18-trihydroxyoctadecanoic acid and 9,10-dihydroxyoctadecanedioic acid.

Glycerol is a major component of suberin, ${ }^{\mathbf{1 0 , 1 7 , 5 2 , 5 3}}$ however its high water solubility justifies that the quantities detected by GC-MS, either before or after alkaline hydrolysis of suberin, were very low (Table 1). Most glycerol released from suberin, due to extraction with cholinium hexanoate, becomes solubilised in water during the filtration and the precipitation steps. After alkaline hydrolysis the hydrolysed suberin fraction is recovered by dichloromethane/water partition, consequently most of the hydrolysed glycerol remains in the aqueous phase. In fact, the total glycerol obtained herein is comparable to that reported for conventionally extracted suberin (ca. 6 wt $\%$, Table 1). ${ }^{52,53}$

Suberin depolymerisation with cholinium hexanoate released increasing quantities of glycerol, from 21.7 to 65.7 $\mathrm{mg}_{\text {glycerol }} / \mathrm{g}_{\text {suberin }}\left(\right.$ glycerol ${ }_{[\mathrm{ChHex}]}$, Table 1$)$. On the contrary, the glycerol released from suberin during alkaline hydrolysis progressively decreases to zero along the extraction time (glycerol $_{[\mathrm{NaOH}]}$, Table 1). This means that after eight hours, virtually all hydrolysable acylglycerol ester bonds present in suberin were cleaved in the ionic liquid media. However, significant amounts of suberin monomers were still released after alkaline hydrolysis. Both ester bonds present in suberin, viz. linear aliphatic and acylglycerol, are labile to the alkaline 


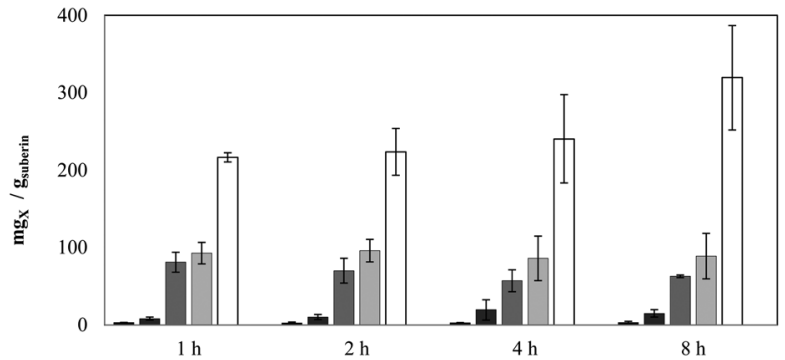

-alkan-1-ols $\square$ alkanoic acids $\square$ phenolics $\square \alpha, \omega$-alkanedioic acids $\square \omega$-hydroxyalkanoic acids

Fig. 3 Quantification of suberin monomers in the samples recovered after depolymerisation in cholinium hexanoate media and submitted to alkaline hydrolysis prior to the GC-MS analysis (GC-MS Method 2). Detailed monomeric quantification in Table S1. $\dagger$

hydrolysis. Thus, it seems that the ionic liquid is efficient towards acylglycerol ester bonds, but fails to cleave most of the linear aliphatic esters bonds.

Suberin solubility in dichloromethane and its recalcitrancy to alkaline hydrolysis increased over the extraction time, from 46.2 to $65.2 \mathrm{wt} \%$ and from 34.4 to $46.3 \mathrm{wt} \%$, respectively for samples extracted for two and eight hours (Table 1). Upon rapid removal from the cell wall to the ionic liquid media, the suberin oligomeric structures undergo continuous depolymerisation hence solubility in dichloromethane increases. At the same time, non-hydrolysable suberin oligomeric structures are progressively removed from the cell wall - hence recalcitrance increases. All aforementioned data was integrated to propose a model for suberin extraction from cork in cholinium hexanoate media (Fig. 4). In brief, after one hour most suberin oligomeric structures, except few non-hydrolysable ones, were removed from the cell wall to the ionic media. Along the eight hours of extraction, suberin oligomeric structures in the ionic liquid media undergo further depolymerisation and glycerol is continuously released. In addition, few non-hydrolysable suberin oligomeric structures were also removed from the cell wall to the ionic liquid media. After eight hours of extraction, linear aliphatic ester bonds were still preserved but most acylglycerol ones were cleaved.

\section{Preferential cleavage of acylglycerol esters in standard compounds}

Apparently cholinium hexanoate promoted efficient cleavage of the acylglycerol ester bonds in suberin, but not of the linear aliphatic ester bonds (see computational study below). To further support these findings, the ionic liquid ability to cleave standard compounds carrying linear aliphatic or acylglycerol ester bonds was analysed by monitoring along time the reaction of glyceryl trioctanoate or octyl octanoate, respectively (Fig. 1). These standard compounds showed different reaction kinetics (Fig. 5). Octyl octanoate was fairly resistant to cleavage. After eight hours of reaction only $c a .30 \mathrm{wt} \%$ was hydrolysed to octanoic acid and octanol. On the contrary, after the same reaction time, more than $80 \mathrm{wt} \%$ of the glyceryl trioctanoate was cleaved, releasing glycerol, octanoic acid and the corresponding diacyl- and monoacylglycerols (Fig. 5). Based on the low amounts of 2-monoacylglycerol released, it is likely that the most favourable cleavage occurred in the acyl group at position C2 of glycerol. This is suggestive of possible steric hindrance and/or chemical selectivity in the reaction promoted by the ionic liquid. This partially explains why amongst the few monomers identified by GC-MS in the suberin samples extracted with cholinium hexanoate none was a 2-monoacylglycerol. ${ }^{32,34}$
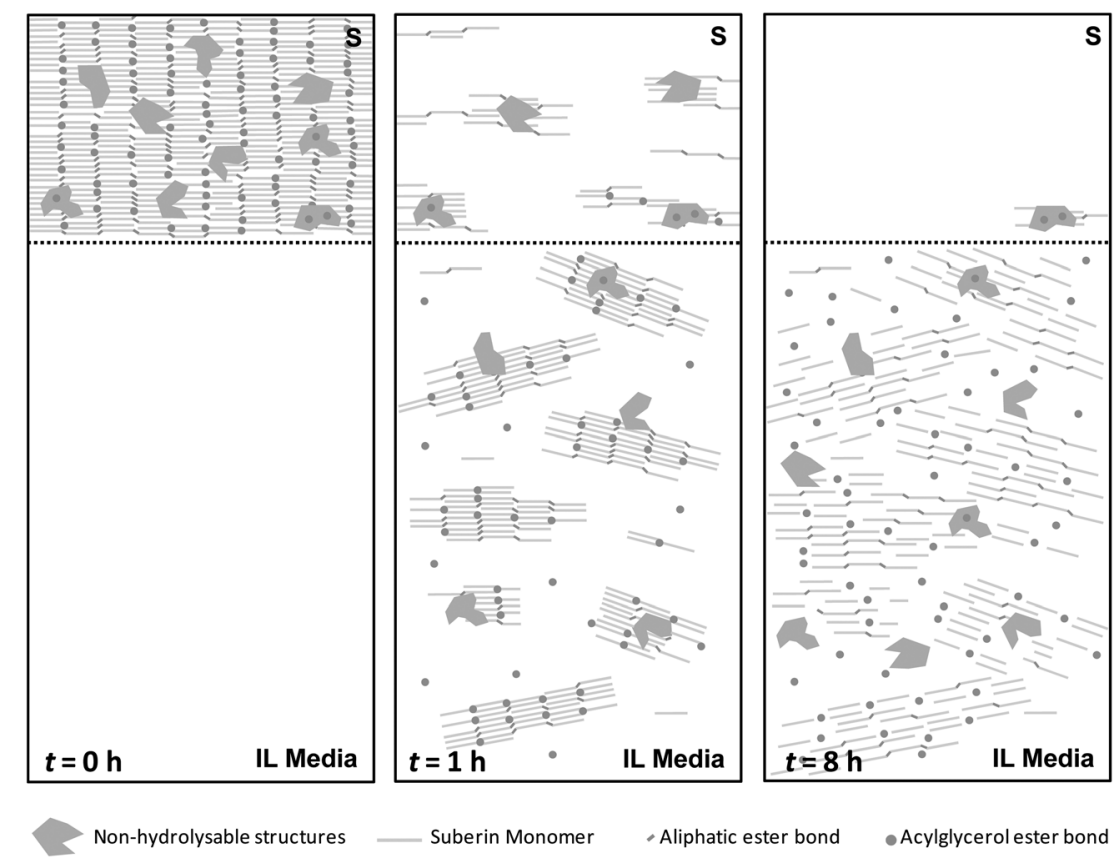

Fig. 4 Schematic view of how cholinium hexanoate leads to suberin depolymerisation along the extraction time. S, suberin deposited in the cell wall; IL Media, Ionic liquid media. 


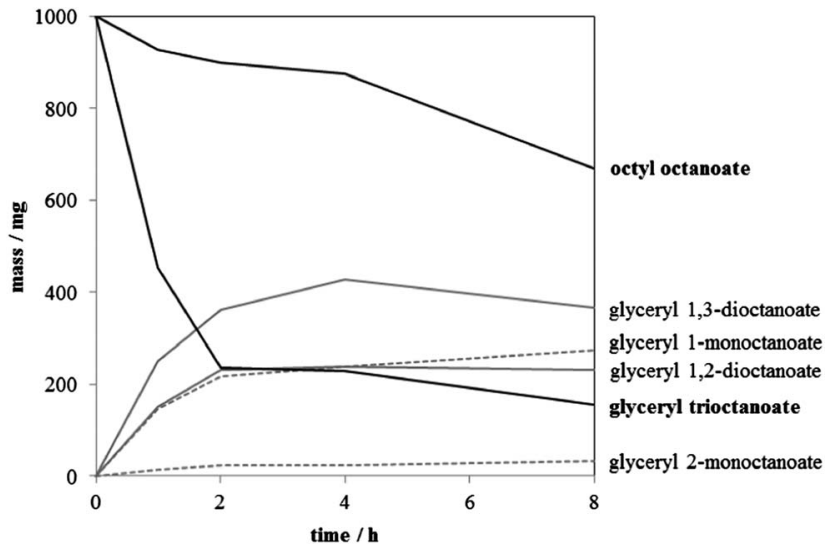

Fig. 5 Compounds detected after treatment of glyceryl trioctanoate and octyl octanoate with cholinium hexanoate during 1, 2, 4 and 8 hours. All compounds were identified and quantified by GC-MS. Glycerol, octanol, octanoic acid and error bars were not represented for ease of visualisation (average standard error $27.8 \mathrm{mg}$ ). At time zero, glyceryl trioctanoate and octyloctanoate were assumed to represent the only compounds present in the mixture.

The lack of efficiency of cholinium hexanoate to promote cleavage of linear aliphatic esters was further confirmed using linear aliphatic polyesters. After four hours, no alterations were detected in the ATR-FTIR spectra of poly(lactic acid) (Fig. S2 $\dagger$ ), in particular in the peaks assigned to $\mathrm{C}=\mathrm{O}$ stretching. Moreover, after this reaction time the mass of the poly(lactic acid) recovered was $\mathrm{ca} .91 .61 \pm 0.26 \mathrm{wt} \%$, agreeing with the amount of lactic acid detected $(6.03 \pm 0.63 \mathrm{wt} \%)$. The ATR-FTIR spectra of poly(12-hydroxydodecanoic acid) (Fig. S3†) was also unaltered after four hours in the ionic liquid media.

Since the epoxy ring is a significant non-structural functionality in suberin its lability in the ionic liquid media was also preliminary analysed using 9,10-epoxy-18-hydroxyoctadecanoic acid. NMR analysis showed that while the epoxy ring was partially preserved after 4 hours, some secondary alcohol signals were also detected $\left({ }^{1} \mathrm{H}\right.$ : $3.5 \mathrm{ppm} ;{ }^{13} \mathrm{C}$ : $72 \mathrm{ppm}$, data not shown). This observation, which was also preliminarily confirmed by GC-MS and ESI-MS (data not shown), can be associated with the opening of the epoxy ring into a vic-diol and/or methoxyhydrin, as suggested above by the ${ }^{13} \mathrm{C} \mathrm{CP} / \mathrm{MAS}$ NMR data of suberin samples.

Suberin is a structural component of the cork cell wall, thus cannot be removed without impairing its integrity (Fig. S1†). The other major polymers in the cork cell wall are likely to be also exposed to the ionic liquid media, namely polysaccharides and lignin, which are linked via ether bonds and ether/C-C bonds, respectively. Preliminary data obtained with $\alpha$-cellulose and alkali lignin, suggested that cholinium hexanoate was unable to promote hydrolysis of their inter-monomeric bonds (data not shown). This further supports cholinium hexanoate selectivity towards suberin..$^{32}$

\section{The role of cholinium hexanoate as catalyst in the hydrolysis of ester bonds}

The cleavage of ester bonds promoted by cholinium hexanoate led to the release of free alcohols and carboxylic acids from glyceryl trioctanoate and octyl octanoate (Fig. 5 and S4 $\dagger$ ), as well as from suberin ${ }^{32,34}$ (GC-MS Method 1, data not shown). This brings further light to this process: the ester bonds were hydrolysed, implying that the ionic liquid plays the dual role of solvent and catalyst (see computational study below). Water was present in the reaction media, since it was ca. $0.2 \mathrm{wt} \%$ in cholinium hexanoate and ca. $2.4 \mathrm{wt} \%$ in cork (determined previously by thermogravimetric analyses). ${ }^{34}$ Thus the molar fraction of water was $4.9 \%$ relative to the ionic liquid $(0.42 \mathrm{wt} \%$ in the reaction media and $8.4 \mathrm{wt} \%$ relative to suberin).

While the capacity of ionic liquids to play the dual role of solvent and catalyst has been well described, ${ }^{54}$ the complex set of chemical and physical interactions, including Coulombic and dipole forces, hydrogen-bonding and acid-base interactions, behind the augmented reactivity in an ionic liquid media are not yet fully understood. Up to now, few hydrolysis reactions in ionic liquid media have been reported, associated to the nucleophilic activation of water. ${ }^{54,55}$ It has been demonstrated that, when present at low concentrations, water is dispersed in the ionic liquid as single molecules. ${ }^{54,55}$ They form specific electrostatic interactions and hydrogen-bonding with the ions. As the concentration of water increases, self-association of the water molecules and ion clustering probably occurs, weakening the catalytic effect of the ionic liquid. The chemistry of water in these domains is still a puzzling scientific question.

It was demonstrated that the hydrolysis kinetics is influenced by the ionic liquid hydrogen bonding donor and acceptor ability. ${ }^{56}$ Cholinium carboxylates are good hydrogen bond acceptors (Kamlet Taft parameters $\beta>0.9) .{ }^{57}$ Accordingly, the mechanism of suberin hydrolysis in cholinium hexanoate media might be related to the nucleophilic activation of water. In fact, increasing the molar fraction of water in the reaction media from $4.9 \%$ to $40 \%$, led to a dramatic reduction in the extraction efficiency of cholinium hexanoate (from $51.0 \pm 5.8$ to $25.3 \pm 2.4 \mathrm{wt} \%$ ). Water in excess hampered the ionic liquid catalytic effect and/or acted as an anti-solvent. Hence, during suberin depolymerisation from cork, a fine balance of the water concentration in the reaction media needs to be attained.

\section{Cholinium hexanoate catalyses the hydrolysis of esters: computational study}

According to the experimental results, cholinium hexanoate catalysed the hydrolysis of esters originating acids and alcohols. A density functional theory (DFT) study $^{\mathbf{4 2 , 4 3}}$ was performed in order to determine the reaction mechanism. All the intermediates and transitions states (TSs) along several possible reaction pathways were calculated (S3†), and the lowest energy pathway obtained, shown in Scheme 1, consists of two steps. In the first step, the hydroxyl group of the cholinium cation $\left(\mathrm{IL}^{+}\right)$ establishes one hydrogen bond with the oxygen of the carbonyl group of the ester, favouring the nucleophilic attack of water at the carbon (TS-1). The intermediate 2a has the newly formed $\mathrm{OH}$ bond. In the second step, one proton is transferred from $\mathbf{2 b}$ to the $\mathrm{O}\left(\mathrm{R}^{2}\right)$ atom, weakening the $\mathrm{C}-\mathrm{O}$ bond (TS-2). The 


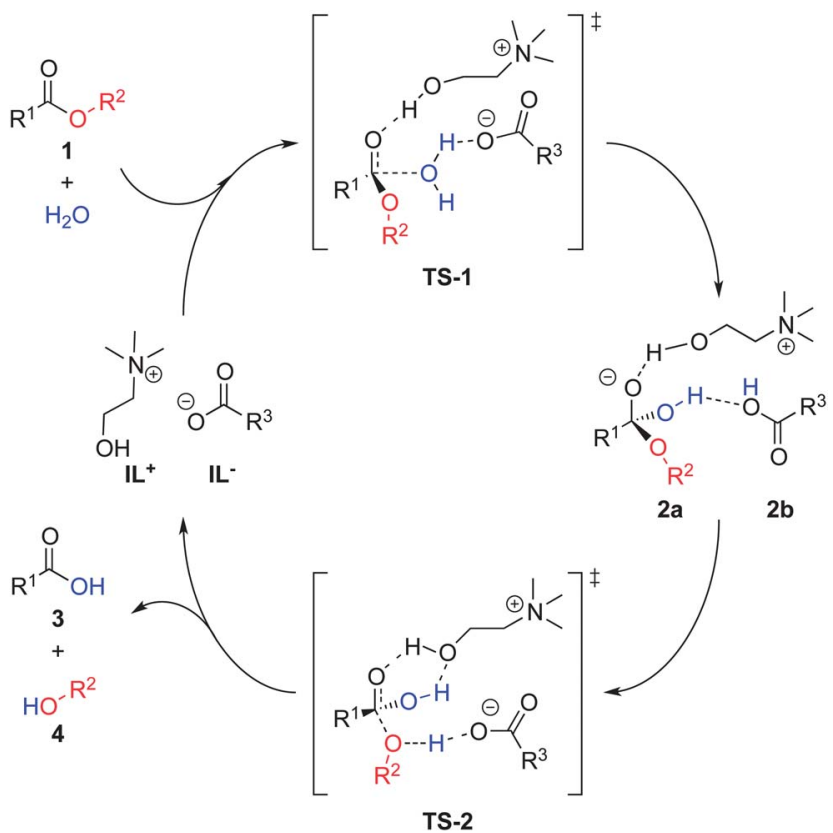

Scheme 1 Proposed mechanism for the hydrolysis of esters catalysed by cholinium alkanoates. TS and IL stand for transition state and ionic liquid, respectively.

products, the acid $\mathbf{3}$ and the alcohol $\mathbf{4}$, are formed and the ionic liquid regenerated, closing the catalytic cycle.

The activation barriers obtained for the hydrolysis of four types of linear aliphatic esters (methyl and ethyl acetate, butyl butyrate, and octyl octanoate) catalysed by cholinium acetate or hexanoate are depicted in Table 2 and Fig. 6 , and show that the second step of the reaction is the limiting one. The energy difference between TS-1 and TS-2 ( $\Delta$ (TS-2-TS-1)) increases with the size of the ester carbon chain, as the linear ester model becomes closer to reality. This arises essentially from the destabilisation of TS-2, since the close proximity of the carbon chain $\mathrm{R}^{1}$ and $\mathrm{R}^{2}$ increases the steric repulsion and thus the activation energy. The calculated activation energies are higher in the reaction catalysed by cholinium hexanoate than by cholinium acetate, reflecting the slightly higher $\mathrm{p} K_{\mathrm{a}}$ of hexanoic acid (4.88vs. 4.76 of acetic acid). This difference is, however, too small and not significant considering the nature of the model, as the substrates are linear aliphatic esters and the trends may easily be reversed. Since cholinium hexanoate efficiency towards the extraction of suberin was 1.5 fold higher than that
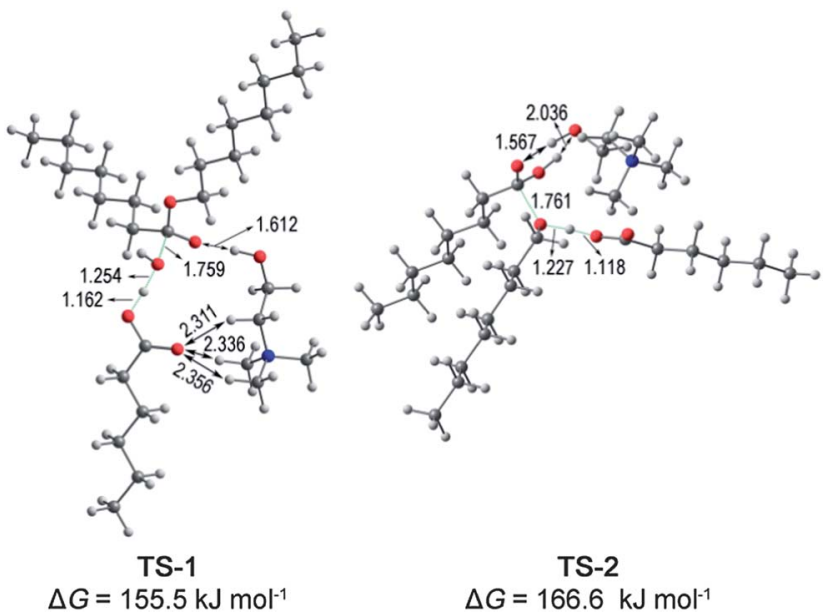

Fig. 6 Calculated transition states (TSs) structures for the two steps of the hydrolysis of octyl octanoate catalysed by cholinium hexanoate. All bond lengths are in angstrom $(\AA)$.

of cholinium acetate, ${ }^{33}$ this is most probably due to the cholinium hexanoate superior solvent ability towards aliphatic chains. This further emphasises the dual role of the cholinium hexanoate, both as solvent and as catalyst.

The experimental results also showed that the hydrolysis catalysed by cholinium hexanoate was faster in triacylglycerol esters than in monoacylglycerol esters (Fig. 5), and that triacylglycerol esters were preferential hydrolysed at the $\mathrm{C} 2$ position (Table 3).

The reaction mechanism was studied considering the hydrolysis of glyceryl triacetate, catalysed by cholinium

Table 3 Gibbs activation energies $\left(\mathrm{kJ} \mathrm{mol}^{1}\right.$ ) calculated for the hydrolysis of glyceryl triacetate catalysed by cholinium acetate

\begin{tabular}{llc}
\hline & & \\
& & \\
\cline { 2 - 3 } & & \\
Transition state & Attack A (C1) & Attack B (C2) \\
\hline TS 1 & 147.8 & 144.3 \\
TS 2 & 151.8 & 146.5 \\
$\Delta$ (TS 2 TS 1) & 4.0 & 2.2
\end{tabular}

Table 2 Gibbs activation energies ( $\left(\mathrm{JJ} \mathrm{mol}{ }^{1}\right.$ ) calculated for the transition states (TSs) presented in Scheme 1

\begin{tabular}{|c|c|c|c|c|c|}
\hline \multirow[b]{2}{*}{ Ionic liquid } & \multirow[b]{2}{*}{ Transition state } & \multicolumn{4}{|c|}{ Ester } \\
\hline & & Methyl acetate & Ethyl acetate & Butyl butyrate & Octyl octanoate \\
\hline & TS 2 & 151.9 & 157.4 & 160.5 & 163.2 \\
\hline & $\Delta($ TS 2 TS 1) & 0.3 & 3.7 & 5.7 & 9.1 \\
\hline Cholinium hexanoate & TS 1 & 153.6 & 154.3 & 156.4 & 155.5 \\
\hline
\end{tabular}


acetate, at both positions $\mathrm{C} 1$ and $\mathrm{C} 2$ (Table 3 ). The second step (TS-2) is again the limiting one for the reaction at both carbon centres. In addition, the reaction at $\mathrm{C} 2$ is faster than at $\mathrm{C} 1$ (lower activation energies), in agreement with the experimental results (Fig. 5).

Finally, the activation energies for the hydrolysis of glyceryl triacetate catalysed by cholinium acetate (Table 3) are lower than those of the linear aliphatic esters (Table 2). This can be correlated to the higher electrophilicity of the ester oxygen atom in the triacylglycerol than in the linear aliphatic ester. These data suggest how cholinium hexanoate might promote a mild depolymerisation of suberin from cork.

\section{Conclusions}

Two distinct phases describe the extraction of suberin from cork with cholinium hexanoate. First, rapid removal of nearly whole suberin, still highly polymerised and cross-linked, occurs. This is followed by slow removal of recalcitrant structures, non-hydrolysable and displaying a high cross-link density, concomitantly with continuous depolymerisation of labile oligomers dispersed in the ionic liquid media (Fig. 4). The ionic liquid plays the dual role of solvent and catalyst and leads to suberin efficient extraction through a mild depolymerisation. Disruption of the threedimensional network of suberin in the ionic liquid media, progresses by preferential cleavage of acylglycerol ester bonds (with the faster kinetics in C2), whilst the linear aliphatic ester bonds remain largely preserved. This reaction mechanism leads to the efficient recovery of a high cross-linked and thus esterified suberin, thus different from that extracted with conventional methods which promote thorough ester cleavage. The ionic liquid catalyses the hydrolysis of esters, hence the water concentration in the reaction media is a decisive parameter. At high concentrations, water weakens the catalytic effect of cholinium hexanoate and/or acts as anti-solvent. A DFT study of the reaction mechanism showed that cholinium hexanoate catalyses the nucleophilic attack of water in two steps. This work should inspire the isolation of biopolyesters from numerous renewable resources, aiming at the development of strategies for their valorisation. A new chemical reaction for the hydrolysis of lipids catalysed by ionic liquids is now available. Ionic liquid chemistry opens the possibility to achieve designer solvents of high performance displaying high selectivity towards a specific carbon centre in an ester bond.

\section{Notes and references}

1 A. Gandini, Green Chem., 2011, 13, 1061-1083.

2 P. E. Kolattukudy, Annu. Rev. Plant Physiol., 1981, 32, 539-567.

3 A. Heredia, Biochim. Biophys. Acta, 2003, 1620, 1-7.

4 K. Ranathunge, L. Schreiber and R. Franke, Plant Science, 2011, 180, 399-413.

5 L. Schreiber, Trends Plant Sci., 2010, 15, 546-553.

6 M. Pollard, F. Beisson, Y. Li and J. Ohlrogge, Trends Plant Sci., 2008, 13, 236-246.

7 F. Beisson, Y. Li-Beisson and M. Pollard, Curr. Opin. Plant Biol., 2012, 15, 329-337.
8 M. A. Bernards, Can. J. Bot., 2002, 80, 227-240.

9 E. C. Lulai and D. L. Corsini, Physiol. Mol. Plant P., 1998, 53(4), 209-222.

10 H. Pereira, Cork: Biology, Production and Uses, Elsevier, Amesterdam, 1st edn, 2007.

11 R. Franke, I. Dombrink and L. Schreiber, Front. Plant Sci., 2012, 3, 1-8.

12 P. E. Kolattukudy and V. P. Agrawal, Lipids, 1974, 9, 682-691.

13 R. Riley and P. E. Kolattukudy, Plant Physiol., 1975, 56, 650654.

14 P. E. Kolattukudy, Science, 1980, 208, 990-1000.

15 M. A. Bernards, in Encyclopedia of Life Sciences, John Wiley \& Sons, Ltd, Chichester, 2002, http://www.els.net.

16 M. A. Bernards, Can. J. Bot., 2002, 80, 227-240.

17 A. Gandini, C. Pascoal and A. J. D. Silvestre, Prog. Polym. Sci., 2006, 31, 878-892.

18 J. Graça and S. Santos, Macromol. Biosci., 2007, 7, 128-135. 19 P. Sitte, Protoplasma, 1962, 54, 555-559.

20 R. E. Stark and J. R. Garbow, Macromolecules, 1992, 25, 149154.

21 M. Lopes, A. Sarychev, C. Neto and A. Gil, Solid State Nucl. Magn. Reson., 2000, 16, 109-121.

22 M. A. Bernards and N. G. Lewis, Phytochemistry, 1998, 47, 915-933.

23 M. A. Bernards, M. L. Lopez, J. Zajicek and N. G. Lewis, J. Biol. Chem., 1995, 270, 7382-7386.

24 J. Negrel, B. Pollet and C. Lapierre, Phytochemistry, 1996, 43, 1195-1199.

25 J. Graça, Phytochem. Rev., 2010, 9, 85-91.

26 N. Cordeiro, M. N. Belgacem, A. Gandini and C. Pascoal Neto, Ind. Crops Prod., 1999, 10, 1-10.

27 N. Cordeiro, A. Blayo, M. N. Belgacem, A. Gandini, C. Pascoal Neto and J. F. LeNest, Ind. Crops Prod., 2000, 11, 63-71.

28 A. F. Sousa, G. Alessandro, A. J. D. Silvestre, C. P. Neto, J. J. C. C. Pinto, C. Eckerman and B. Holmbom, J. Polym. Sci., Part A: Polym. Chem., 2011, 49, 2281-2291.

29 A. Sousa, A. Gandini, A. Silvestre and C. Pascoal Neto, ChemSusChem, 2008, 1, 1020-1025.

30 R. Ekman and C. Eckerman, Pap. Ja Puu-Pap. Timber, 1985, 67, 255-273.

31 M. H. Lopes, A. M. Gil, A. J. D. Silvestre and C. P. Neto, J. Agric. Food Chem., 2000, 48, 383-391.

32 R. Ferreira, H. Garcia, A. F. Sousa, M. Petkovic, P. Lamosa, C. S. R. Freire, A. J. D. Silvestre, L. P. N. Rebelo and C. Silva Pereira, New J. Chem., 2012, 36, 2014-2024.

33 H. Garcia, R. Ferreira, M. Petkovic, J. L. Ferguson, M. C. Leitão, H. Q. N. Gunaratne, K. R. Seddon, L. P. N. Rebelo and C. Silva Pereira, Green Chem., 2010, 12, 367-369.

34 R. Ferreira, H. Garcia, A. F. Sousa, C. S. R. Freire, A. J. D. Silvestre, L. P. N. Rebelo and C. Silva Pereira, Ind. Crops Prod., 2013, 44, 520-527.

35 M. Petkovic, J. L. Ferguson, H. Q. N. Gunaratne, R. Ferreira, M. C. Leitão, K. R. Seddon, L. P. N. Rebelo and C. Silva Pereira, Green Chem., 2010, 12, 643-649.

36 A. M. Gil, M. Lopes, J. Rocha and C. P. Neto, Int. J. Biol. Macromol., 1997, 20, 293-305. 
37 A. Olsson, M. Lindström and T. Iversen, Biomacromolecules, 2007, 8, 757-760.

38 N. Cordeiro, M. N. Belgacem, A. J. D. Silvestre, C. P. Neto and A. Gandini, Int. J. Biol. Macromol., 1998, 22, 71-80.

39 P. C. R. O. Pinto, A. R. Sousa, A. J. D. Silvestre, C. P. Neto, A. Gandini, C. Eckerman and B. Holmbom, Ind. Crops Prod., 2009, 29, 126-132.

40 R. Ekman, Holzforschung, 1983, 37, 205-211.

41 A. F. Sousa, P. C. R. O. Pinto, A. J. D. Silvestre and C. P. Neto, J. Agric. Food Chem., 2006, 54, 6888-6893.

$42 \mathrm{~W}$. Koch and M. C. Holthausen, A chemist's guide to density functional theory, Wiley-VCH, New York, 2nd edn, 2001.

43 R. G. Parr and W. Yang, Density-functional theory of atoms and molecules, Oxford University Press, Oxford, 1989.

44 M. J. Frisch, G. W. Trucks, H. B. Schlegel, G. E. Scuseria, M. A. Robb, J. R. Cheeseman, G. Scalmani, V. Barone, B. Mennucci, G. A. Petersson, H. Nakatsuji, M. Caricato, X. Li, H. P. Hratchian, A. F. Izmaylov, J. Bloino, G. Zheng, J. L. Sonnenberg, M. Hada, M. Ehara, K. Toyota, R. Fukuda, J. Hasegawa, M. Ishida, T. Nakajima, Y. Honda, O. Kitao, H. Nakai, T. Vreven, J. A. Montgomery, Jr, J. E. Peralta, F. Ogliaro, M. Bearpark, J. J. Heyd, E. Brothers, K. N. Kudin, V. N. Staroverov, R. Kobayashi, J. Normand, K. Raghavachari, A. Rendell, J. C. Burant, S. S. Iyengar, J. Tomasi, M. Cossi, N. Rega, J. M. Millam, M. Klene, J. E. Knox, J. B. Cross, V. Bakken, C. Adamo, J. Jaramillo, R. Gomperts, R. E. Stratmann, O. Yazyev, A. J. Austin, R. Cammi, C. Pomelli, J. W. Ochterski,
R. L. Martin, K. Morokuma, V. G. Zakrzewski, G. A. Voth, P. Salvador, J. J. Dannenberg, S. Dapprich, A. D. Daniels, O. Farkas, J. B. Foresman, J. V. Ortiz, J. Cioslowski and D. J. Fox, Gaussian 09, Revision B. 1, Gaussian, Wallingford, CT, 2009.

45 J. P. Perdew, K. Burke and M. Ernzerhof, Phys. Rev. Lett., 1996, 77, 3865-3868.

46 J. P. Perdew, K. Burke and M. Ernzerhof, Phys. Rev. Lett., 1997, 78, 1396.

47 C. Adamo and V. Barone, J. Chem. Phys., 1999, 110, 61586170.

48 S. E. Wheeler, A. Moran, S. N. Pieniazek and K. N. Houk, J. Phys. Chem. A, 2009, 113, 10376-10384.

49 J. Tomasi, B. Mennucci and R. Cammi, Chem. Rev., 2005, 105, 2999-3093.

$50 \mathrm{~J}$. W. Turner, B. E. Hartman and P. G. Hatcher, Org. Geochem., 2013, 57, 41-53.

51 A. P. Deshmukh, A. J. Simpson, C. M. Hadad and P. G. Hatcher, Org. Geochem., 2005, 36, 1072-1085.

52 J. Graça and H. Pereira, Holzforschung, 1997, 51, 225-234.

53 H. Pereira, BioResources, 2013, 8, 2246-2256.

54 J. Hallett and T. Welton, Chem. Rev., 2011, 111, 3508-3576.

55 S. Chowdhury, R. S. Mohan and J. L. Scott, Tetrahedron, 2007, 63, 2663-2389.

56 C. Weber, A. Masters and T. Maschmeyer, J. Phys. Chem. B, 2012, 116, 1858-1864.

57 J. Restolho, J. L. Mata and B. Saramago, Fluid Phase Equilib., 2012, 322-323, 142-147. 